\title{
Erosion et types de cavitation
}

\author{
Erosion and cavitation types
}

\author{
Yves Lecoffre \\ Professeur associé à l'ENSGI Grenoble \\ YLec Consultants \\ Allée des Dauphins, 38330 Saint-Ismier - Tél. : (33) 76521863
}

\section{Introduction}

L'érosion de cavitation résulte de l'accumulation des impacts produits par les collapses de structures de vapeur près d'une paroi solide. Cette agression se traduit, au début du phénomène, par l'apparition de petites indentations dans le matériau, sans perte de masse, puis, à mesure que le nombre d'impacts augmente, par des dégâts macroscopiques pouvant, dans certains cas, conduire à des dysfonctionnements des composants, ou même à des destructions irréversibles et dangereuses.

Les récents développements sur les lois de similitude caractérisant ces phénomènes laissent espérer que l'on disposera dans un avenir proche de techniques de prédiction des dégâts au réel à partir d'essais réalisés sur modèle. La mise en œuvre de ces techniques reste néanmoins assez lourde et il serait souhaitable que l'on dispose, au stade du bureau d'études, de moyens de prédiction basés sur des approches moins sophistiquées.

C'est dans cet esprit que nous avions effectué une recherche exploratoire sur l'agressivité des écoulements cavitants en fonction de la dimension des structures de vapeur et des gradients de pression qu'elles rencontrent dans les zones de collapse. Cette analyse était partiellement confortée par les résultats déjà acquis sur des écoulements géométriquement semblables.

\section{Méthode d'approche}

\subsection{L'intensité d'une cavité de vapeur}

Dans les recherches antérieures menées chez NEYRTEC, nous avions défini l'intensité I d'une cavité de vapeur comme sa propension à créer une marque identifiable dans un matériau à partir d'une certaine vitesse d'écoulement.
On a montré que cette intensité était fonction de la nature du matériau et du fluide utilisé, mais était indépendante de la dimension générale de l'écoulement. On a proposé des lois de similitude tenant compte de tous ces aspects, dont l'essentiel a été vérifié expérimentalement (réf. 1). Ces résultats permettent d'espérer la mise au point d'une méthode de prédiction des dégâts au réel à partir d'essais sur modèle de courte durée. La vérification de la faisabilité de cette procédure ferait actuellement l'objet d'un programme de recherche européen.

Une méthode de prédiction, sans doute moins précise, mais qui permettrait d'évaluer les risques dès le stade du bureau d'études, pourrait prendre en compte certains de ces résultats en insistant sur l'influence des gradients de pression et des types de cavitation sur les risques d'érosion.

L'intensité d'une cavité individuelle tendra à augmenter lorsque sa dimension initiale sera plus importante ou que le gradient de pression qu'elle rencontre sera plus intense. Les impacts produiront des marques d'autant plus importantes que la dimension de ces cavités sera elle-même importante.

Au niveau d'un écoulement, qui engendre de nombreuses structures de vapeur, les dégâts à attendre seront, bien entendu, directement fonction de la fréquence de production des cavités.

Les dégâts macroscopiques à terme résulteront donc, au premier ordre, de ces trois paramètres, la dimension initiale des cavités, le gradient de pression dans la zone de collapse et la productivité des écoulements.

\subsection{Formalisme}

\section{Principes}

On suppose que les cavités de vapeur sont toutes assimilables à des bulles sphériques. On suppose que, lors de leur 


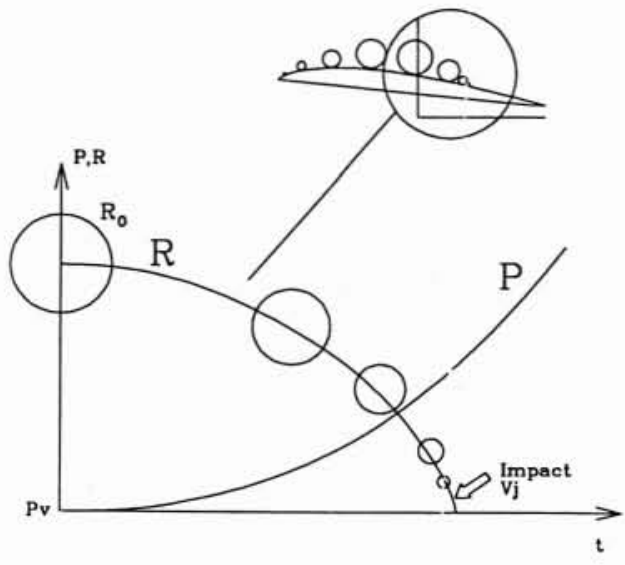

1. Evolution de la pression et du rayon de la cavité dans la zone de collapse. Vitesse d'impact.

collapse, ces bulles ont une même forme pour une réduction donnée de leur volume. Cette forme, à volume réduit donné peut être identifiée par la présence d'un jet rentrant ou de toute autre particularité géométrique. L'important est que l'on puisse déterminer une vitesse caractéristique des parois $V_{j}$ à l'instant de l'impact (fig. 1).

On a montré dans les études en référence 1 ou 2 que l'intensité d'une cavité n'était fonction que de $\rho_{c} C V_{j}$ étant la vitesse caractéristique des parois de la bulle à l'instant de l'impact, $\rho_{c}$ étant l'impédance acoustique du fluide considéré.

Il en résulte que la simple connaissance de $V_{j}$, pour un fluide donné, détermine l'intensité d'une cavité.

Si l'on suppose maintenant que les vitesses de collapse sont complètement déterminées par l'application de l'équation de Rayleigh-Plesset, la valeur de $V_{j}$ est la vitesse de la paroi de la bulle pour une valeur donnée de son volume réduit, probablement bien supérieure à celle pour laquelle les effets de compressibilité se font sentir (réf. 2).

Si l'on connaît la dimension initiale de la bulle et le gradient de pression auquel elle va être soumise, il suffit de calculer le collapse par application de l'équation de RAYLEIGH-Plesset (réf. 3 ).

On se donne alors un critère pour déterminer le volume réduit pour lequel se produit le choc, par exemple $1 / 100 \mathrm{du}$ volume initial de la bulle. On détermine ainsi, connaissant une vitesse $V_{j 0}$ critique dans un cas de référence, la vitesse d'apparition de l'érosion dans le cas considéré.

\section{Utilisation de lois puissance}

Pour simplifier l'approche, nous avons supposé que le collapse d'une structure de vapeur quelconque se produisait dans un champ de pression dont l'évolution temporelle était de la forme :

$$
P(t)-P_{v}=k t^{n}
$$

Le rayon initial de la bulle avant collapse est donné par $R_{0}$.

On applique l'équation de RAYLEIGH-PLESSET en supprimant les termes secondaires faisant intervenir la viscosité et la tension superficielle ainsi que la compressibilité du gaz. Pour ce dernier terme, on a pu montrer expérimentalement (réf. 2) qu'il n'avait pas d'influence dans une situation industrielle courante. Un calcul d'ordre de grandeur montre que les deux premiers sont également secondaires dans la phase de collapse.

$$
R R^{\prime \prime}+3 / 2 R^{\prime} 2=\left(P_{v}-P\right) / \rho .
$$

On utilise les grandeurs réduites

$$
\begin{aligned}
r & =R / R_{0} \\
\tau & =t / T_{0} .
\end{aligned}
$$

$R_{0}$ et $T_{0}$ étant respectivement un rayon et un temps caractéristiques du collapse.

L'équation de RAYLEIGH-PLeSSET s'écrit alors :

$$
\rho R_{0}^{2} / T_{0}^{2}\left(r r^{\prime \prime}+3 / 2 r^{\prime} 2\right)=k \tau^{n} T_{o}^{n} .
$$

Cette équation est universelle pour peu que

$$
\rho R_{0}^{2}=k T_{0}^{(\mathrm{n}+2)} .
$$

Soit

$$
T_{\mathrm{o}}=\left(r R_{0}^{2} / k\right)^{1 /(n+2)} .
$$

On en déduit la vitesse d'impact

$$
V_{j} \sim R_{\mathrm{o}} / T_{0}=\left(k / \rho R_{0}^{\mathrm{n}}\right)^{1 /(n+2)} .
$$

Dans un champ de pression caractérisé par $\Delta P=k t^{n}$, la vitesse de paroi des bulles est proportionnelle à $k^{1 /(n+2)}$ et $R_{0}^{\mathrm{n} /(\mathrm{n}+2)}$

$$
V_{j} \sim k^{1 /(n+2)} R_{0}^{\mathrm{n} /(\mathrm{n}+2)}
$$

pour

$$
\Delta P=k t^{n} .
$$

En appliquant cette relation, on retrouve tous les invariants classiques caractérisant le collapse des bulles, et, en particulier le fait que les vitesses $V_{j}$ de collapse pour deux bulles homothétiques dans deux écoulements semblables ne dépendent que du $\sigma$ de cavitation et de la vitesse d'écoulement.

Cette approche met en évidence l'influence combinée de la variation temporelle de pression vue par les bulles et de leur rayon initial.

En particulier, pour un palier brutal de pression, correspondant à $n=0$, la vitesse de collapse est indépendante du diamètre initial des bulles. Au contraire, dans le cas limite d'une montée en pression correspondant à $n$ infini, la vitesse de collapse deviendrait proportionnelle au rayon initial. 


\section{Applications}

\subsection{Principes}

Il était intéressant d'appliquer les résultats théoriques cidessus à des cas d'écoulement eux-mêmes théoriques. Aussi avons-nous choisi deux géométries très simplistes, le cône emboîté et le dièdre.

On a supposé que les vitesses dans toute section étaient homogènes et que l'épaisseur de la poche de vapeur était faible par rapport au jeu annulaire ou à la distance entre les plans du dièdre. On a enfin supposé que, lorsque se produisait une cavitation, sous forme d'une poche de vapeur, les vitesses dans l'un ou l'autre des passages étaient constantes sur toute la longueur de la poche. Ceci résulte du fait que la pression dans la poche étant constante, la vitesse à l'interface, par application de l'équation de Bernoulli l'est également.

Ces hypothèses, extrêmement grossières, n'ont pour objectif que de permettre d'effectuer un calcul illustrant les conséquences de l'approche ci-dessus.

On a donc déterminé, dans un premier temps, les gradients de pression, puis, en fonction des vitesses et du développement des poches de cavitation, l'évolution temporelle des pressions dans la zone de collapse.

On a supposé que la dimension initiale $R_{0}$ des cavités de vapeur était égale à l'épaisseur de la poche de vapeur dans la zone de collapse.

On a donc pu en déduire des vitesses adimensionnelles d'impact $V_{j}$ par application de la formule ci-dessus.

\subsection{Résultats de calcul}

Les figures 2 et 3 donnent les résultats obtenus dans le cas d'un cône et d'un dièdre de hauteur initiale égale à $20 \mathrm{~mm}$. $x$ est la longueur de la cavité.

On constate que l'intensité des cavités, leur propension, rappelons-le, à produire une marque permanente dans un matériau, passe, pour une vitesse donnée par un maximum fonction de l'angle du cône ou du dièdre (fig. 2).

Bien entendu, pour une longueur de poche donnée, la vitesse d'interface est proportionnelle à la vitesse d'écoulement, et l'intensité est d'autant plus importante que celleci est élevée (fig. 2). Ceci résulte directement des règles de similitude hydrodynamique.

Les résultats montrent également de manière évidente que la vitesse d'écoulement n'est pas le seul paramètre caractéristique des risques d'érosion, les cavités produites par certains écoulements à $20 \mathrm{~m} / \mathrm{s}$ étant aussi intenses que d'autres à $60 \mathrm{~m} / \mathrm{s}$. C'est, par exemple le cas des points A et $\mathrm{B}$ de la figure 2 .

Enfin, cette approche met en évidence que les écoulements ne sont pas d'autant plus agressifs que la cavitation est développée, mais qu'il existe un maximum, correspondant à un $\sigma$ critique, pour lequel le couple rayon initial, gradient de pression maximalise les vitesses réduites d'impact. Ce maximum d'intensité se produit pour des longueurs de poches d'autant plus courtes que le gradient de pression est intense (fig. 3).

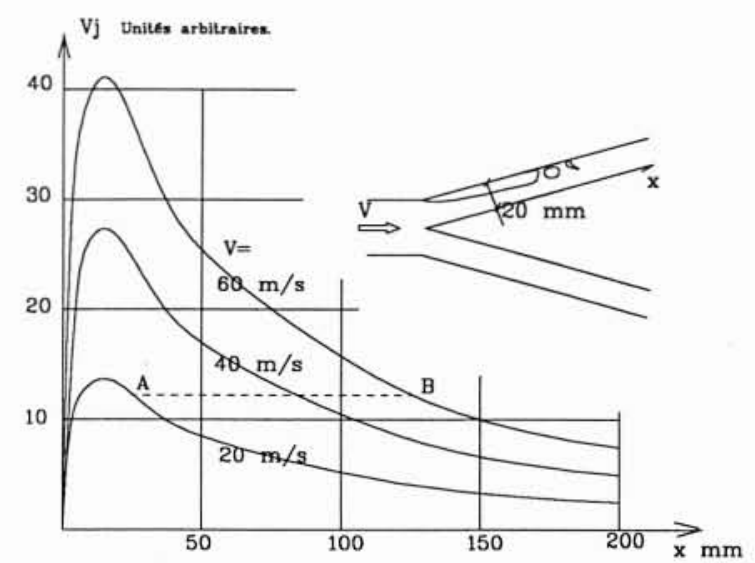

2. Vitesses d'impact en fonction de la longueur de la poche de vapeur. Cas du cône.

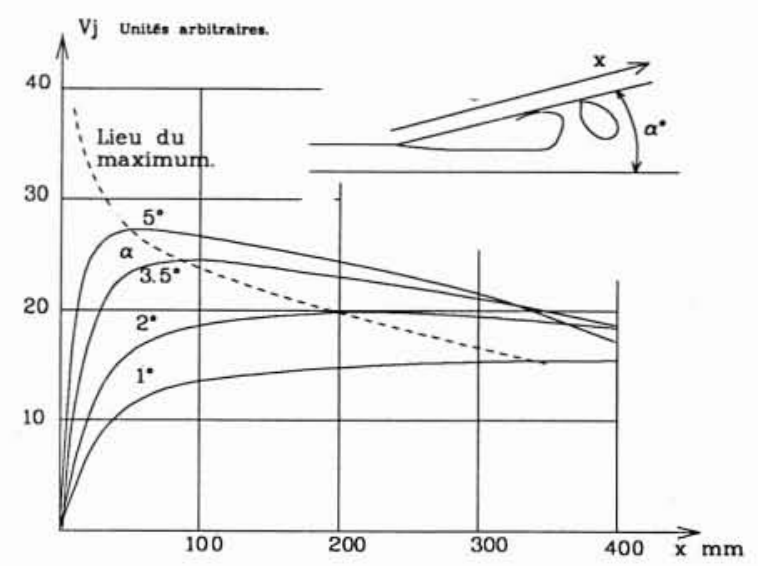

3. Vitesses d'impact en fonction de l'angle $\alpha$. Cas du dièdre.

\section{Expériences}

\subsection{Méthodes de dépouillement}

Les expériences sont difficiles à quantifier de manière précise, car la marque d'un écoulement sur un matériau est caractérisée par un histogramme et donc par une distribution d'impacts. A l'époque où ces expériences ont été menées, chez NEYRTEC, en 1983, nous ne disposions pas des méthodes optiques automatisées de mesure des histogrammes par interférométrie. Nous devions nous contenter, pour valider ces approches, d'observations visuelles sous microscope par méthode d'interférométrie différentielle Nomarski.

Les comparaisons ont donc été faites de manière qualitative, par analyse comparée de clichés. 

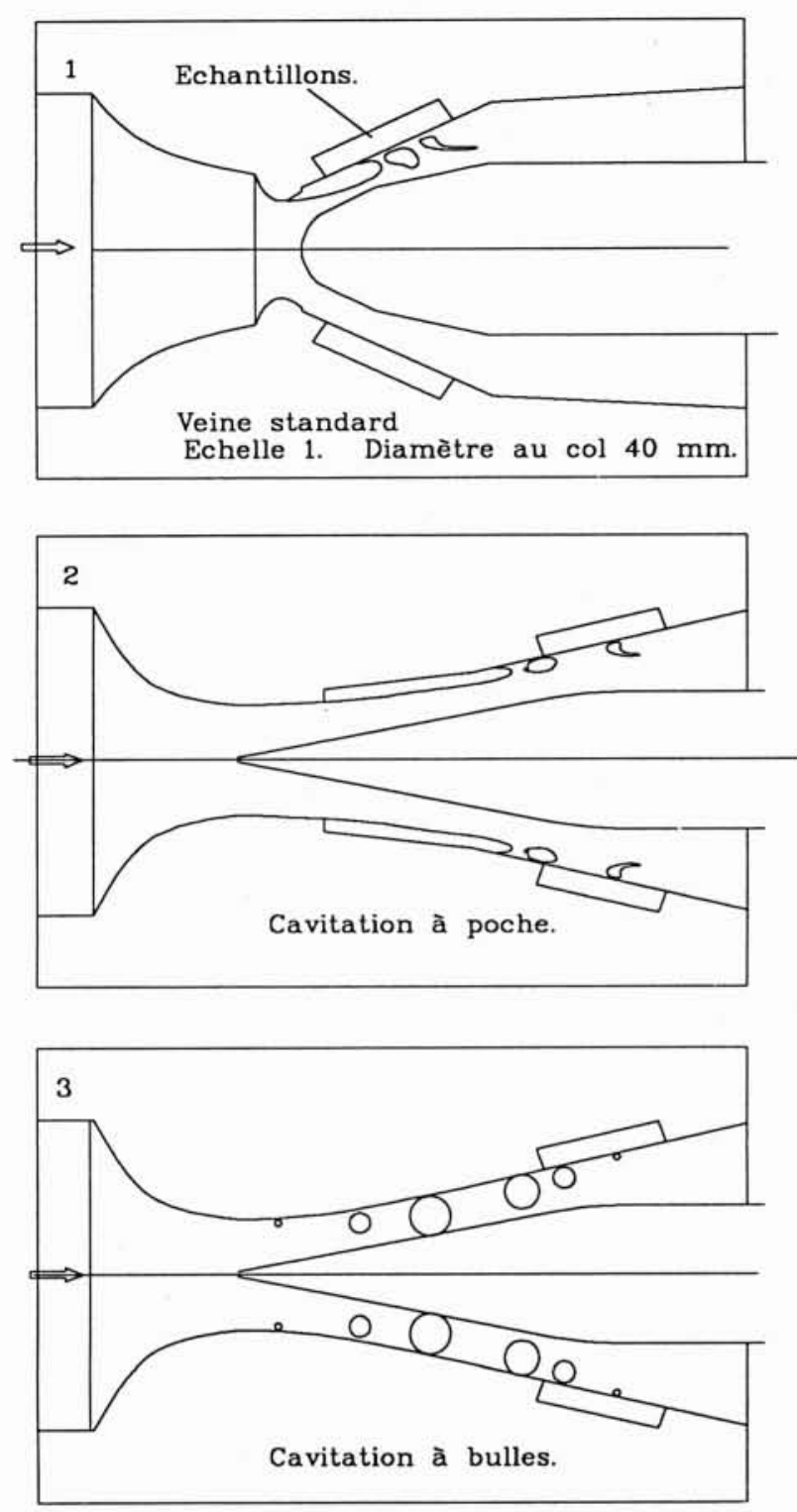

4. Veines d'essais.

\subsection{Ecoulements}

La géométrie d'écoulement utilisée est assimilable à celle d'un cône emboîté. Trois veines d'essais ont été réalisées, l'une d'elles (veine 3) présentant des cavitations à bulles, les deux autres ( 1 et 2 ) des cavitations à poche. Toutes ces veines avaient un diamètre au col d'environ $40 \mathrm{~mm}$.

\section{Cavitation à poche}

Les angles au sommet des cônes des veines 1 et 2 ont été respectivement de $25^{\circ}$ et $14^{\circ}$; l'angle de $25^{\circ}$ conduisant à un gradient de pression plus important que celui de $14^{\circ}$ dans la zone de collapse pour une poche de longueur donnée.

\section{Comparaison bulles-poche}

L'une des veines, (3) d'angle au sommet $14^{\circ}$, présentait des parois continues à faible rayon de courbure permettant d'engendrer une cavitation à bulles. Une autre, de géométrie autrement identique, était munie d'un décrochement local permettant de produire une cavitation à poche (fig. 2 et 3).

On disposait enfin d'une injection de germes permettant de considérer l'influence du nombre de bulles sur l'érosion produite dans la veine à paroi continue.

\section{Tunnel hydrodynamique}

Les expériences ont été menées sur le tunnel THV de NEYRTEC, capable d'àtteindre des vitesses de $60 \mathrm{~m} / \mathrm{s}$ au débit maximal de $600 \mathrm{l} / \mathrm{s}$.

\subsection{Résultats}

Sans entrer dans les détails des résultats, comme nous l'avons dit assez difficiles à analyser lorsqu'on ne dispose pas de méthodes de mesure des histogrammes, on a pu effectuer les constatations suivantes :

\section{Influence du type de cavitation}

La cavitation à bulles est beaucoup plus agressive que la cavitation à poche pour une géométrie d'écoulement, et donc de gradient de pression dans la zone de collapse, données. La raison en est probablement que les bulles sont beaucoup plus grosses que les cavités engendrées par les poches, et que, par conséquent, leurs vitesses caractéristiques $V_{j}$ sont, toutes choses égales par ailleurs, supérieures. La photographie $\mathrm{n}^{\circ} 1$ montre les impacts produits par une cavitation à bulles ou à poche à même $\sigma(0,60)$ et même vitesse d'écoulement $(30 \mathrm{~m} / \mathrm{s})$ sur des échantillons d'acier inoxydable.

Notons, pour l'anecdote, qu'alors que nous avons effectué des essais de cavitation à poches jusqu'à des vitesses de $60 \mathrm{~m} / \mathrm{s}$ dans la veine standard dont le gradient de pression est très élevé, nous hésitions à dépasser des vitesses de $30 \mathrm{~m} / \mathrm{s}$ dans le tunnel en raison de la violence des impacts produits dans les conditions de cavitation à bulles.

\section{Influence du gradient de pression}

La photographie $\mathrm{n}^{\circ} 2$ montre les impacts produits sur de l'acier inoxydable par une cavitation à poche dans la veine (1) d'angle $25^{\circ}$ et celle (2) d'angle $14^{\circ}$ pour une même longueur de poche de cavitation et une même vitesse d'écoulement $(30 \mathrm{~m} / \mathrm{s})$.

Il est frappant de comparer les dimensions des impacts obtenus dans les deux cas. Ceci confirme l'influence primordiale du gradient de pression. (En fait, gradient de pression et dimension des cavités sont combinés dans cet essai et leurs effets s'ajoutent.)

Ces résultats sont en accord avec les conclusions de la théorie sommaire exposée ci-dessus. Ils confirment l'importance des deux paramètres que nous avons analysés. 


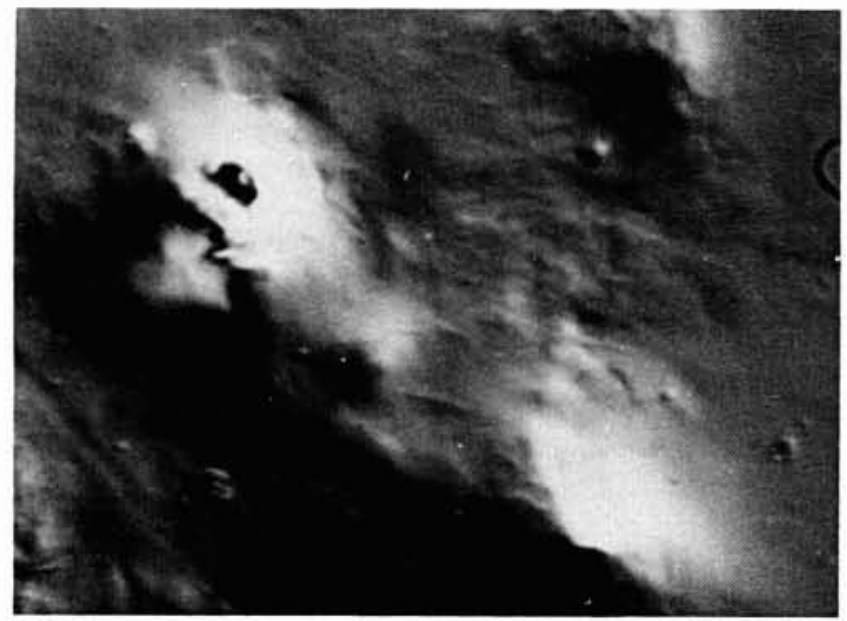

Bulles

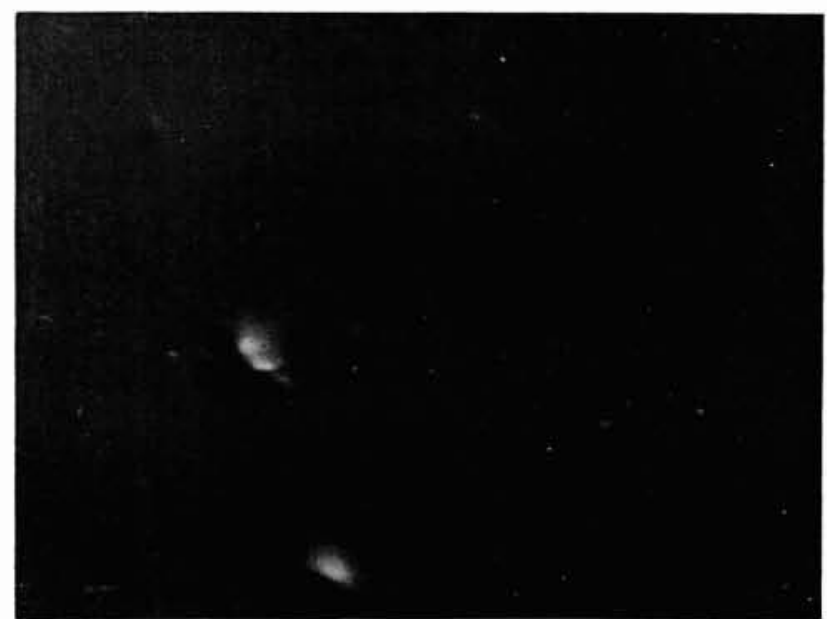

Poche.

Photographie $\mathbf{n}^{\circ} \mathbf{1}$. Impacts produits par une cavitation à bulles et à poche dans les veines 3 et 2 . $V=30 \mathrm{~m} / \mathrm{s}$. Grandissement $\times 95$.

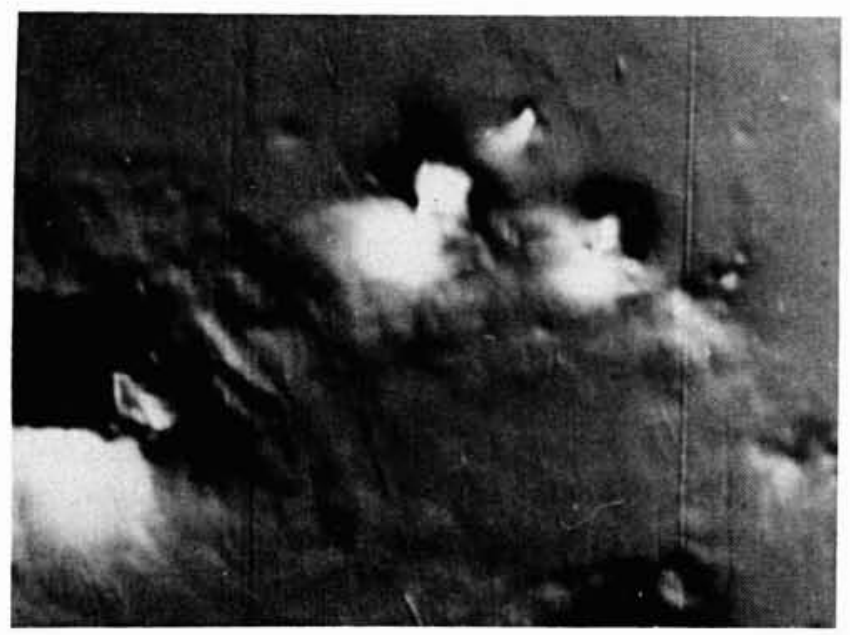

Veine $1,25^{\circ}$

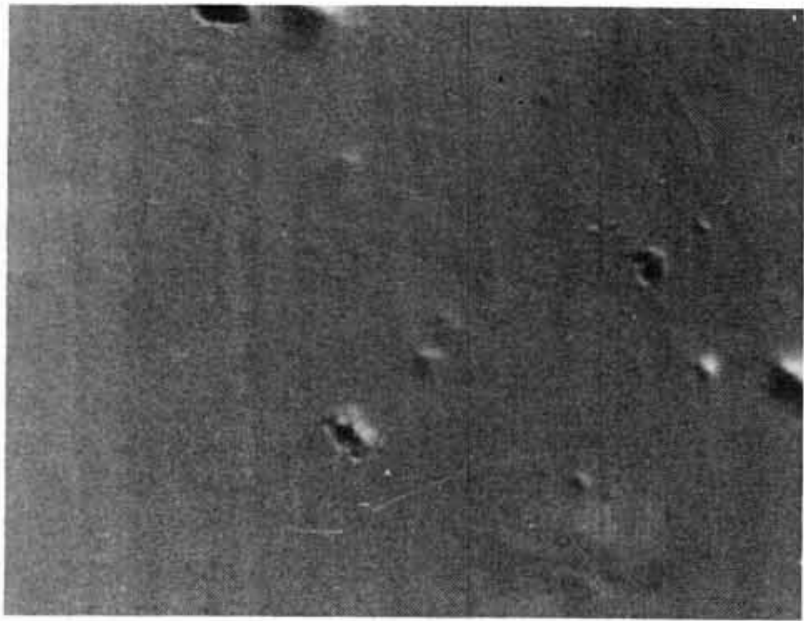

Veine $2,14^{\circ}$.

Photographie $\mathbf{n}^{\circ} \mathbf{2}$. Impacts produits par des cavitations à poche dans les veines $l$ et 2 . $V=30 \mathrm{~m} / \mathrm{s}$. Grandissement $\times 95$.

\section{Interprétation des résultats}

\subsection{Types de cavitation}

Dans les conditions d'essais que nous avons réalisés, la cavitation à bulles est plus agressive que la cavitation à poches. Il serait cependant dangereux de généraliser sans analyser plus en détail le comportement des machines industrielles.

En général, on admet dans une machine un certain développement de la cavitation, qui se traduit pratiquement par une longueur admissible de la zone cavitante sur les aubages.

La cavitation à bulles correspond à une situation à faible incidence, dans laquelle les gradients de pression sont réduits. Même si les bulles sont de diamètre important, il se peut que le gradient de pression auquel elles sont soumises ne soit pas suffisant pour créer des érosions importantes.

Au contraire, dans le cas de cavitation à poche, les gradients de pression dans la zone de collapse sont plus élevés, d'autant plus que les incidences sont grandes.

A mesure que l'on augmente les incidences, on risque de créer des dégâts importants par l'effet combiné de l'augmentation des gradients de pression et des épaisseurs de poche.

La prédiction de l'intensité des cavités doit donc tenir compte de tous ces facteurs, et il est fort probable qu'elle passe par un minimum à mesure que l'incidence du profil augmente, ce minimum correspondant, à longueur de cavité donnée, au point de transition entre cavitation à bulles et à poche. 


\section{Y. LECOFFRE}

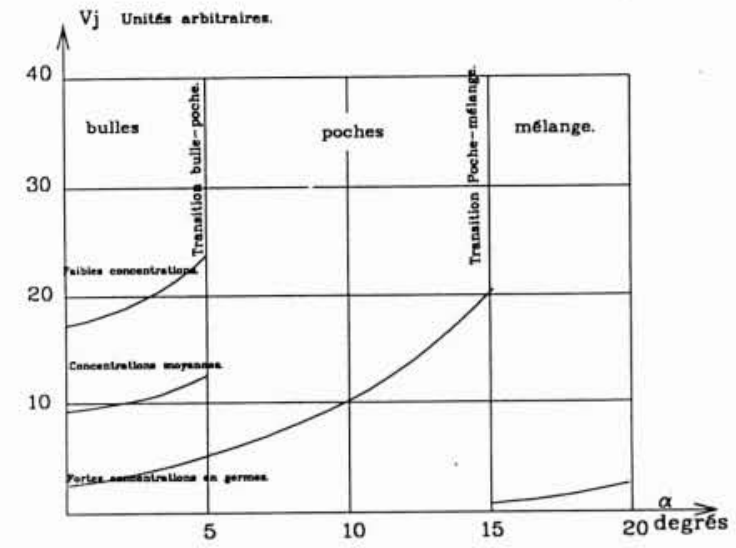

5. Evolution de l'intensité des cavités.

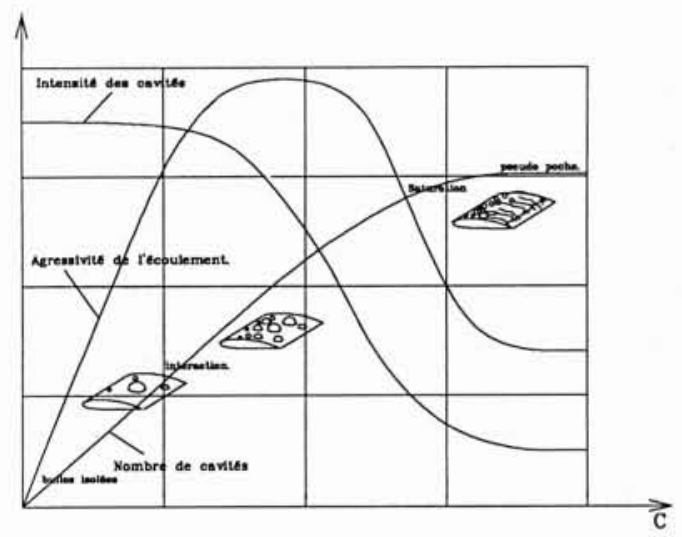

6. Concentration en germes et érosion. Cavitation à bulles.

La figure 5 donne un aperçu qualitatif de l'intensité des cavités lorsqu'on augmente l'incidence d'un profil. En cavitation à bulles, plus le nombre de germes est élevé, et moins les vitesses d'impact sont importantes. Pour de très grandes concentrations en germes, la géométrie de la poche de vapeur tend à se confondre avec celle d'une poche continue (fig. 6). Pour de faibles teneurs en germes, les impacts produits sont très violents, et leur intensité décroît brutalement à la transition entre bulles et poche.

\subsection{Nombre de cavités}

Si l'intensité des cavités individuelles ne dépend pas de leur nombre, l'agressivité de l'écoulement, et donc les dégâts à long terme qu'il risque d'engendrer, est directement proportionnelle à la fréquence de production des cavités.
En cavitation à bulles, ce nombre de cavités est proportionnel au nombre de germes actifs excités par l'écoulement. On pourrait donc penser qu'il suffit de limiter ce nombre de germes pour éviter l'érosion. Il n'en est malheureusement rien. Comme le schématise la figure 6 , pour une géométrie d'écoulement donnée, la dimension des bulles de vapeur diminue. Leur intensité individuelle est donc réduite. Il est, là aussi, probable que les dégâts produits par une cavitation à bulles passent par un maximum à mesure de l'augmentation des concentrations en germes actifs.

En cavitation à poche, le problème est en principe plus simple, puisque l'écoulement est indépendant de la qualité de l'eau. Le nombre de cavités engendrées est proportionnel au nombre de Strouhal et à l'inverse d'une dimension caractéristique de l'écoulement, en l'occurrence la hauteur de poche. Là encore, lorsque l'on augmente l'incidence à vitesse d'écoulement constante, deux phénomènes entrent en conflit: le gradient de pression et la dimension des cavités augmentent, tandis que le nombre de cavités engendrées diminue. Les premiers effets seront, à notre avis, prépondérants, et l'érosion augmentera avec l'incidence, à longueur de poche donnée.

Il serait, bien entendu, nécessaire dans un cas d'application pratique, de calculer les épaisseurs de poches et le gradient de pression dans la zone de collapse pour donner des éléments de réponse exploitables.

La figure 7 donne l'évolution probable de l'agressivité de l'écoulement, à vitesse et longueur de cavités constantes, lorsque l'on fait varier l'incidence du profil. Deux zones nous semblent particulièrement dangereuses, celle qui correspond à la cavitation à bulles avec un nombre de germes peu ou moyennement élevé, et la cavitation à poches courtes à forte incidence.

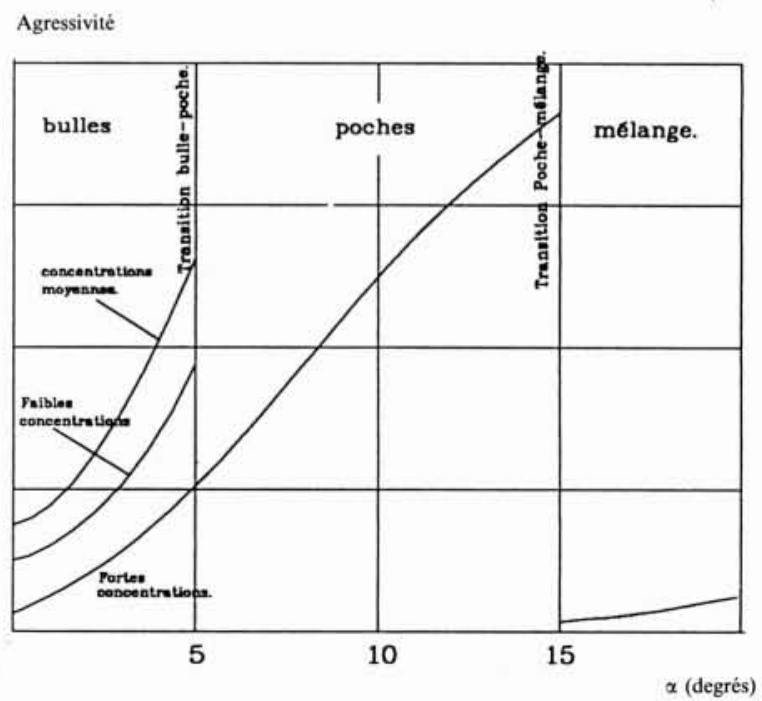

7. Evolution de l'agressivité de l'écoulement. 


\section{Développements envisageables}

\subsection{Modélisation}

Les résultats expérimentaux obtenus, malgré leur caractère semi quantitatif, montrent des tendances claires en ce qui concerne l'intensité des cavités.

Certains logiciels permettent de calculer avec un degré d'approximation raisonnable le développement de la cavitation dans une machine et les gradients de pression dans la zone de fermeture.

En utilisant ces modélisations, dont la qualité va en s'améliorant, et en appliquant des critères de Strouhal, on pourra ainsi avoir une première approche de l'agressivité de l'écoulement.

De telles méthodes ne seraient qu'une adaptation de programmes existants, et seraient donc facilement utilisables par un bureau d'études disposant de ces codes de calcul. Bien entendu, le collapse des bulles serait calculé pour chaque cas pratique par application de l'équation de RAYLEIGH-PLESSET.

\subsection{Expérimental}

Des expériences à caractère quantitatif seraient nécessaires pour juger de l'agressivité réelle des écoulements dans des situations de référence. On devra, en particulier, définir un critère donnant la dimension équivalente des cavités. Il serait intéressant de mesurer des histogrammes d'impacts pour quelques couples de gradients de pression, de types de cavitation et de dimensions de cavités. L'application des règles de similitude permettrait ensuite d'extrapoler ces résultats à d'autres dimensions, d'autres fluides et d'autres matériaux.

Ces quelques essais pourraient être la base d'une banque de données utilisable au stade de la conception.

\section{Conclusion}

Si des campagnes d'essais à échelle 1 ou à base de règles de similitude restent nécessaires pour prévoir les érosions dues à des écoulements cavitants, on peut envisager d'effectuer une première estimation des risques en bureau d'études en utilisant les programmes de calcul de géométrie des écoulements cavitants actuellement disponibles.

La procédure prendrait en compte le développement de la cavitation, les gradients de pression dans la zone de collapse, le type de cavitation et les paramètres de fonctionnement global de la machine, $\sigma$, vitesse de rotation et débit réduit par exemple dans le cas d'une turbomachine.

Il serait nécessaire de s'appuyer sur des essais de référence relativement peu nombreux, car faciles à extrapoler à d'autres conditions expérimentales comparables par application des règles de similitude. Ces essais de base pourraient être menés sur un petit moyen d'essais spécialisé, par exemple une installation Modulab (réf. 4). Il serait alors nécessaire de réaliser systématiquement des histogrammes d'indentations et de mesurer les dégâts à long terme obtenus sur plusieurs matériaux industriels. L'ensemble constituerait une base de données qu'il suffirait d'utiliser dans le cadre d'une procédure automatique de prédiction utilisant les codes de calculs hydrodynamiques adaptés.

\section{Remerciements}

L'étude constituant la base de cet article a été financée par la DRET (Contrat 82/396).

Elle a été réalisée dans le laboratoire de NEYRTEC (Actuellement acb-CERG).

L'auteur remercie acb-CERG pour lui avoir accordé la permission de publier.

\section{Références}

[1] Lecoffre Y., Marcoz J., Franc J.-P., Michel J.-M. Tentative procedure for scaling cavitation damage. ASME int. symp. on cavitation in hydraulic structures and turbomachinery. Albuquerque, June 1985.

[2] Amblard A., Lecoffre Y. - The non effect of dissolved air on cavitation erosion. ASME Winter annual meeting. San Francisco, 1988.

[3] Rayleigh Lord (Strutt, John William). - On the pressures developed in a liquid during the collapse of a spherical cavity. Phil Mag, 34, 95-98, Aug 1917.

[4] Le Modulab, Notice YLec Consultants, SPRETEC. 


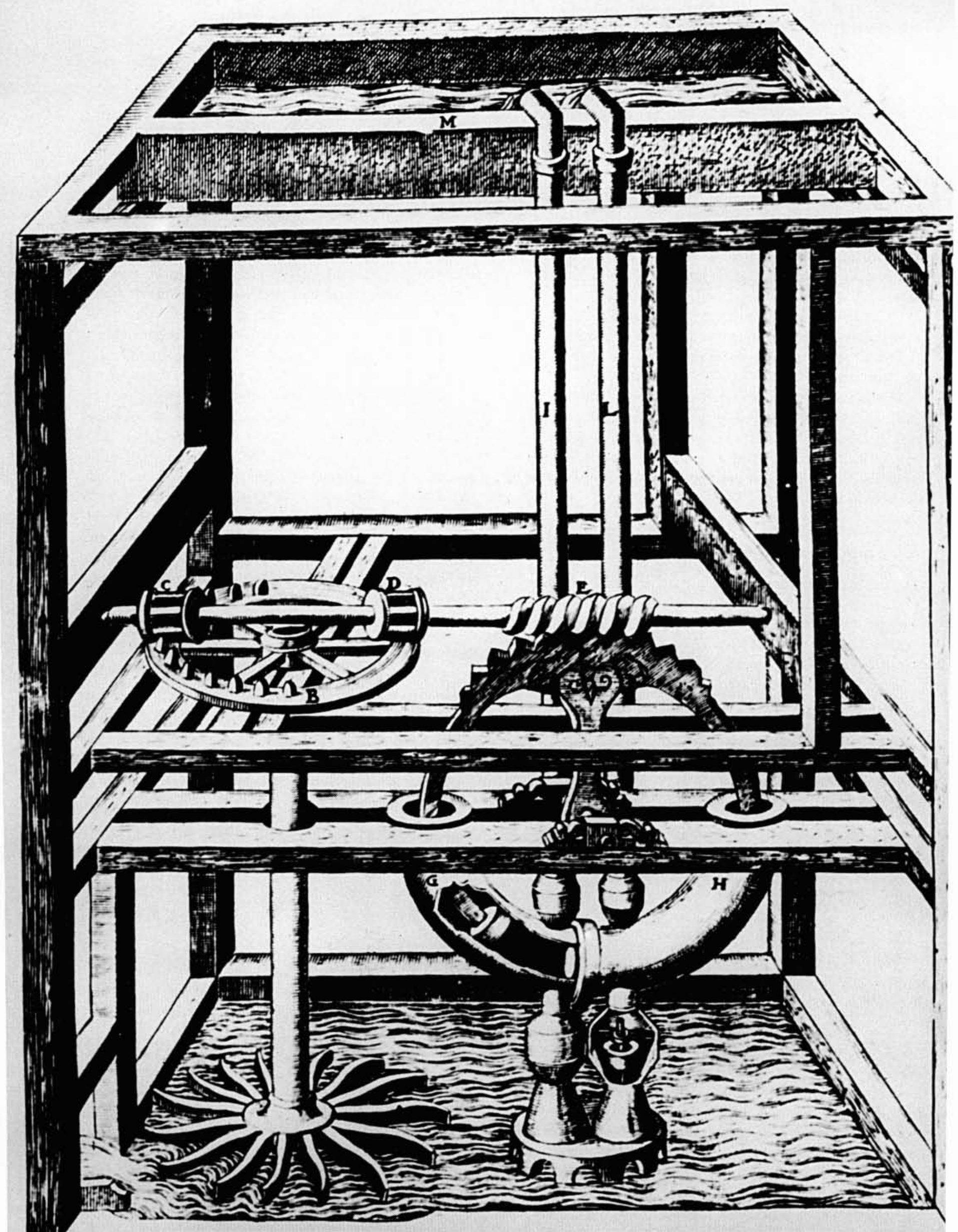

and

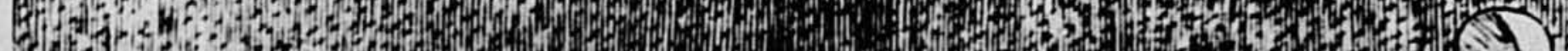

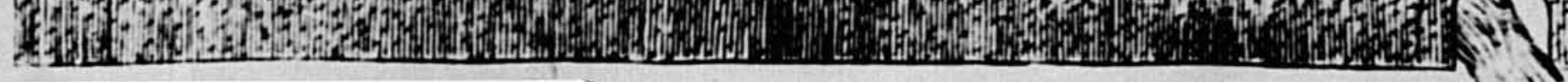

